\title{
Symptoms of Ocular Myasthenia Gravis Masked by an Organic Lesion in Sphenoid Sinuses
}

\author{
Rajeshwary Aroor ${ }^{1}$, Marina Saldanha ${ }^{2}$, Sharik Mustafa ${ }^{3}$, Thayyezhuth Devika ${ }^{4}$
}

\begin{abstract}
Otolaryngologist tends to assume that the cranial nerve palsy is due to local causes, but it is important to overlook the symptoms to rule out other neurological causes too. Here, we present a 79-year-old elderly man, diabetic, who presented to our department with ophthalmoplegia along with contrast-enhanced computed tomography scan of nose and paranasal sinuses did in another hospital showing suspected mass lesion in bilateral sphenoid sinuses with the expansion of the sinus wall and erosion of the posteromedial wall of the right orbit. A provisional diagnosis of ophthalmoplegia secondary to pressure effect from the mass lesion in the sphenoid sinuses was considered. The patient underwent functional endoscopic sinus surgery (FESS) and fungal debris was removed from the sphenoid and ethmoid sinuses on both sides. Immediate postoperative period, he had improvement in ptosis. On subsequent follow-up, there was a worsening of his symptoms and neurological evaluation done. A repetitive nerve conduction study revealed abnormal decrement in the bilateral median and facial nerves. Blood serum levels showed elevated acetylcholine receptor antibody $(10 \mathrm{nmol} / \mathrm{L})$. Thus, a diagnosis of ocular myasthenia gravis was made. The patient was symptomatically better with neostigmine $(60 \mathrm{mg})$ and ophthalmoplegia improved.

Keywords: Fungal sinusitis, Ocular myasthenia, Sphenoid sinusitis.

Clinical Rhinology An International Journal (2020): 10.5005/jp-journals-10013-1369
\end{abstract}

\section{INTRODUCTION}

Isolated sphenoid sinusitis though rare, rhinologists are familiar with this in recent years. Ocular cranial nerve involvement like abducent nerve palsy and oculomotor nerve palsy are uncommon complications of sphenoid sinusitis. ${ }^{1}$ The abducent nerve is commonly involved due to its close medial anatomical location in the cavernous sinus. Most of the time diagnosis of isolated sphenoid sinusitis is an incidental finding in a radiological investigation. Ocular myasthenia gravis is known to cause isolated cranial nerve palsy, ophthalmoplegia leading to ptosis and diplopia. Organic pathology in the sphenoid sinus may mask the clinical presentation of myasthenia gravis leading to diagnostic delay.

\section{Case Description}

A 79-year-old elderly man who is a known diabetic, presented with diplopia and drooping of the right eye of 1-month duration. Ocular examination showed left lateral rectus palsy and right ocular ptosis (Fig. 1). The rest of the ear, nose, and throat examination was normal. Contrast-enhanced computed tomography scan (CT) of the nose and paranasal sinuses which was done in the previous hospital where the patient was initially evaluated for the same problem showed soft tissue density lesion filling the sphenoid and posterior ethmoidal sinus with the erosion of sphenoidal floor, intersphenoidal septum, and posteromedial wall of the right orbit (Fig. 2). In view of these findings, the possibility of mass lesion in the sphenoid sinus probably fungal debris was considered. Magnetic resonance imaging of the brain and orbit was done which showed mild retro-orbital soft tissue hyperintensity on the right side (Fig. 3). The patient underwent endoscopic transethmoidal sphenoidotomy. Bilateral sphenoid sinuses and posterior ethmoidal sinuses were filled with fungal debris which was cleared. In the immediate postoperative period, there was an improvement in the right eye ptosis and ocular movements. The patient was discharged on the second postoperative day. The patient was reported 15 days
1,2,4 Department of Otorhinolaryngology, KS Hegde Medical Academy, Nitte (Deemed to be University), Mangaluru, Karnataka, India

${ }^{3}$ Department of Neurology, KS Hegde Medical Academy, Nitte (Deemed to be University), Mangaluru, Karnataka, India

Corresponding Author: Rajeshwary Aroor, Department of Otorhinolaryngology, KS Hegde Medical Academy, Nitte (Deemed to be University), Mangaluru, Karnataka, India, Phone: +91 9448150448, e-mail: rajeshwarisomayaji@gmail.com

How to cite this article: Aroor R, Saldanha M, Mustafa S, et al. Symptoms of Ocular Myasthenia Gravis Masked by an Organic Lesion in Sphenoid Sinuses. Clin Rhinol An Int J 2020;13(1):18-20.

Source of support: Nil

Conflict of interest: None

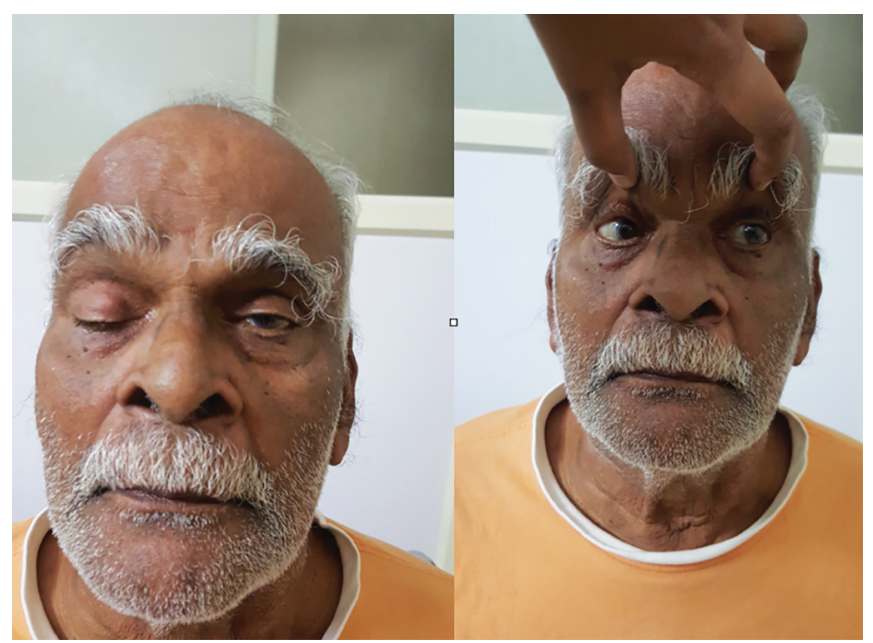

Fig. 1: Showing left lateral rectus palsy and right ptosis

(c) The Author(s). 2020 Open Access This article is distributed under the terms of the Creative Commons Attribution 4.0 International License (https://creativecommons.org/licenses/by-nc/4.0/), which permits unrestricted use, distribution, and non-commercial reproduction in anymedium, provided you give appropriate credit to the original author(s) and the source, provide a link to the Creative Commons license, and indicate if changes were made. The Creative Commons Public Domain Dedication waiver (http://creativecommons.org/publicdomain/zero/1.0/) applies to the data made available in this article, unless otherwise stated. 


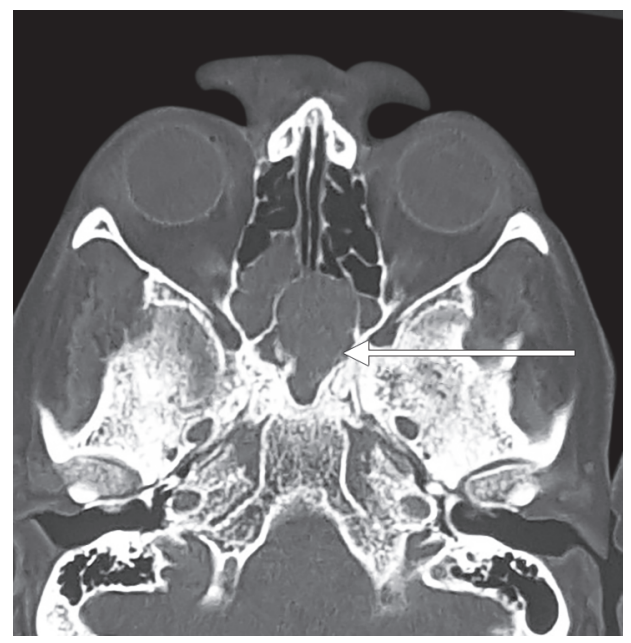

Fig. 2: CT scan showing soft tissue density lesion filling the sphenoid and posterior ethmoidal sinus

after the surgery with the recurrence of the same symptoms. Neurology reference was attained in view of persisting ptosis and lateral rectus palsy after a repeat CT scan of nose and sinus showed a healthy sinus cavity. A repetitive nerve stimulation study showed an abnormal decrement in the bilateral median and facial nerves. Blood serum levels showed elevated acetylcholine receptor antibody (10 nmol/L), which was further confirmed as myasthenia gravis. A contrast-enhanced computed tomography scan of the thorax was normal. The patient was treated with pyridostigmine 60 $\mathrm{mg} /$ day and his ocular palsies recovered and were symptomatically better (Fig. 4).

\section{Discussion}

Sphenoid sinusitis is usually accompanied by the involvement of other sinuses. Isolated sphenoid sinus disease is associated with diagnostic and therapeutic challenges to the surgeon. Involvement of ocular nerves is more common in invasive fungal sinusitis.

The involvement of the abducent nerve secondary to sphenoid sinusitis is explained in the following ways: (1) Direct involvement of nerve sheath. (2) Expansion of the sphenoid sinus into the cavernous sinus or superior orbital fissure and compressing the abducent nerve. (3) Vasculitis or cavernous sinus thrombosis causing ischemic infarction of abducent nerve. ${ }^{2}$ Involvement of dorsal clival artery which supplies the proximal portion of abducent nerve. ${ }^{3}$ Dehiscent lateral wall of sphenoid sinus. ${ }^{4}$ Cranial nerve involvement in sphenoid sinusitis though rare commonly seen in fungal sinusitis.

More than $60 \%$ of myasthenia gravis patient presents with drooping of an eyelid or double vision. ${ }^{5}$ The extraocular muscle weakness of myasthenia gravis is considered as pseudo-internuclear ophthalmoplegia or pseudo third nerve palsy. ${ }^{6}$

Since this patient initially presented to us with a radiological investigation which was suggestive of mass lesions in the sphenoid sinus our initial diagnosis was ophthalmoplegia secondary to pressure effect from the mass lesion in the sphenoid sinuses. The patient and his family members failed to give other important histories suggestive of myasthenia gravis such as easy fatigability, muscle weakness as they were probably thinking that these symptoms were due to his advanced age. Diabetic patients are prone to ptosis and ophthalmoplegia secondary to diabetic

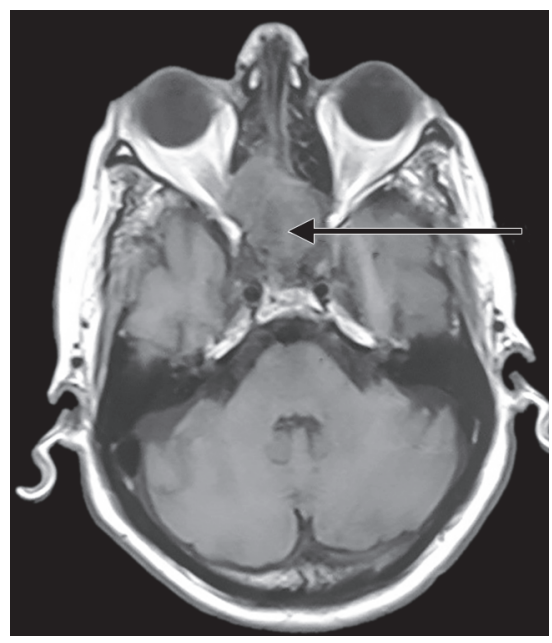

Fig. 3: Magnetic resonance imaging of brain and orbit was done which showed mild retro-orbital soft tissue hyperintensity in right side

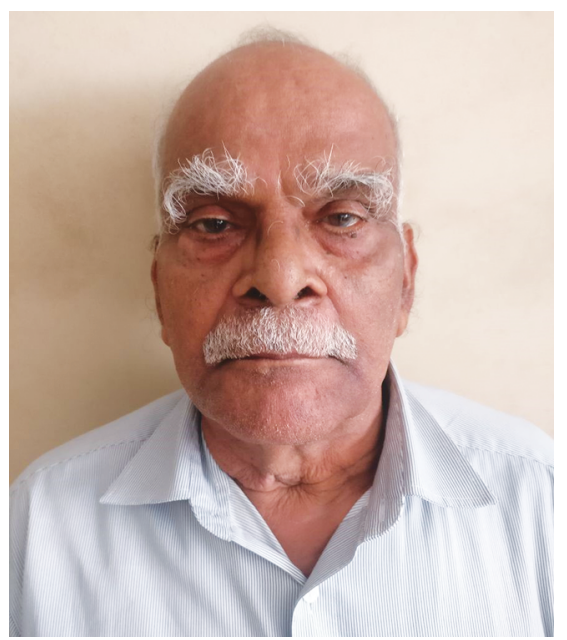

Fig. 4: Ocular palsies recovered after treatment

neuropathy. ${ }^{7}$ Since our patient is also a known diabetic for 10 years, diabetic ophthalmoplegia was also thought of.

Though anesthesia complications are more in patients with undiagnosed myasthenia gravis both our patient and we were lucky that this patient did not develop any untoward anesthetic complications during procedure and reversal. Fentanyl $100 \mu \mathrm{g}$ and propofol $150 \mathrm{mg}$ intravenously were given as induction anesthesia for the endoscopic surgery in this patient. Atracurium $30 \mathrm{mg}$ was given intravenously as a muscle relaxant and anesthesia was maintained with nitrous oxide and isoflurane. The dose of muscle relaxant required for sinus surgery is usually much less compared to other surgeries.

This may be the reason for the uneventful anesthesia period in our patient as surgery was completed with a single dose of atracurium. The patient was reversed with neostigmine $(3.5 \mathrm{mg}$ ) given intravenously. The intraoperative and postoperative period was uneventful which probably because atracurium is a safer drug in myasthenia gravis because of its shorter duration and less cumulative effect at the neuromuscular junction. ${ }^{8}$ The reason for the improvement in patient's ophthalmoplegia in the immediate postoperative period is probably due to neostigmine. 


\section{CONCLUSION}

From this patient, we conclude that a thorough detailed case evaluation has to be done before going into a definite treatment. Though organic pathology is evident, one has to look for all other possible causes as in our case, though fungal ball in the sphenoid sinus was very much evident as the cause for ophthalmoplegia it was not so. The hasty decision in diagnosis and management is disastrous to both the surgeon and the patient.

\section{References}

1. Whan Kim S, Woo Kim D, Gyu Kong I, et al. Isolated sphenoid sinus diseases: report of 76 cases. Acta Otolaryngol 2008;128(4):455-459. DOI: 10.1080/00016480701762466.

2. Patt BS, Manning SC. Blindness resulting from orbital complications of sinusitis. Otolaryngol Head Neck Surg 1991;104(6):789-795. DOI: $10.1177 / 019459989110400604$
3. Krisht A, Barnett DW, Barrow DL, et al. The blood supply of the intracavernous cranial nerves: an anatomic study. Neurosurgery 1994;34(2):275-279. DOI: 10.1227/00006123-199402000-00011.

4. Khattar VS, Hathiram BT, Sharma H. Sternberg's canal and the controversies surrounding it. Otorhinolaryngol Clin 2011;3(3):184-187. DOI: 10.5005/jp-journals-10003-1079.

5. Luchanok U, Kaminski HJ. Ocular myasthenia: diagnostic and treatment recommendations and the evidence base. Curr Opin Neurol 2008;21(1):8-15. DOI: 10.1097/WCO.0b013e3282f4098e.

6. Jay WM, Nazarian SM, Underwood DW. Pseudo-internuclear ophthalmoplegia with downshoot in myasthenia gravis. J Clin Neuroophthalmol 1987;7(2):74-76.

7. Edwards JL, Vincent AM, Cheng HT, et al. Diabetic neuropathy: mechanisms to management. Pharmacol Ther 2008;120(1):1-34. DOI: 10.1016/j.pharmthera.2008.05.005.

8. Ramsey FM, Smith GD. Clinical use of atracurium in myasthenia gravis: a case report. Can Anaesth Soc J 1985;32(6):642-645. DOI: 10.1007/ BF03011412. 